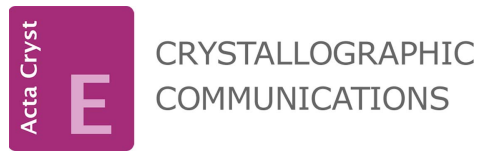

OPEN Ә ACCESS

ISSN 2056-9890

\section{The crystal structure of a new polymorph of hexaaquanickel(II) bis(6-oxo-1,6-di- hydropyridine-3-carboxylate)}

\author{
Rubén Pérez-Aguirre, Sonia Pérez-Yáñez, Garikoitz \\ Beobide, Oscar Castillo, ${ }^{*}$ Juan Manuel Gutiérrez-Zorrilla \\ and Antonio Luque \\ Departamento de Química Inorgánica., Facultad de Ciencia y Tecnología, \\ Universidad del País Vasco, UPV/EHU, Apdo. 644, E-48080 Bilbao, Spain. \\ *Correspondence e-mail: oscar.castillo@ehu.eus
}

Received 16 November 2015; accepted 24 November 2015

Edited by M. Weil, Vienna University of Technology, Austria

In a new polymorph of the title salt, $\left[\mathrm{Ni}\left(\mathrm{H}_{2} \mathrm{O}\right)_{6}\right]\left(\mathrm{C}_{6} \mathrm{H}_{4} \mathrm{NO}_{3}\right)_{2}$, the metal atom of the cationic complex lies on a symmetry centre and is coordinated by six water molecules to provide a quite regular octahedral coordination environment. These cations interact with 6-oxo-1,6-dihydropyridine-3-carboxylate anions through electrostatic interactions and by means of $\mathrm{O}-$ $\mathrm{H} \cdots \mathrm{O}$ and $\mathrm{N}-\mathrm{H} \cdots \mathrm{O}$ hydrogen bonds involving the carboxylate, keto and protonated imine groups of the anion, and the coordinating water molecules from the cationic complex entity to generate a supramolecular three-dimensional architecture. The previously reported polymorph of this compound presents a network of hydrogen bonds, in which the organic anions establish mutual hydrogen-bonding interactions involving their keto and protonated imine groups.

Keywords: crystal structure; polymorph; 6-oxo-1,6-dihydropyridine-3carboxylate anion; hydrogen bonding,

CCDC reference: 1438522

\section{Related literature}

The zinc and cobalt analogues (Zhang et al., 2005; Song et al., 2005; Zhang \& Ng, 2005a) of the title salt are isostructural with the previously reported polymorph of $\left[\mathrm{Ni}\left(\mathrm{H}_{2} \mathrm{O}\right)_{6}\right]\left(\mathrm{C}_{6} \mathrm{H}_{4} \mathrm{NO}_{3}\right)_{2}$ (Zhang \& Ng, 2005b). It is worth mentioning that although the authors claimed a lactim tautomer of the organic anion to be present in all these structures, the $\mathrm{C}-\mathrm{O}$ bond length seems to indicate of a lactam tautomer as in the case of the title compound. For additional examples of coordination complexes with 6-oxo-1,6-dihydropyridine-3-carboxylate anions and copper(II), see: Zeng et al. (2007).

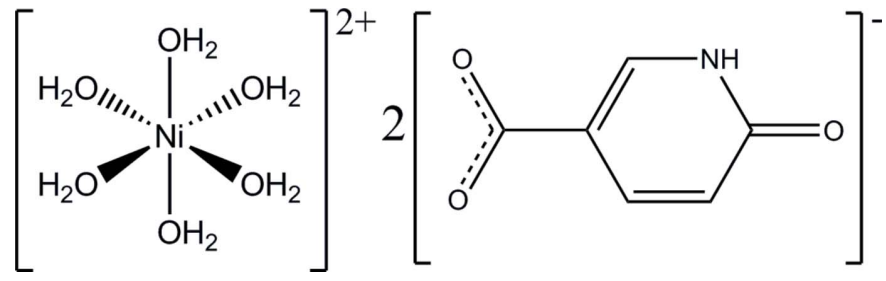

\section{Experimental}

2.1. Crystal data

$\left[\mathrm{Ni}\left(\mathrm{H}_{2} \mathrm{O}\right)_{6}\right]\left(\mathrm{C}_{6} \mathrm{H}_{4} \mathrm{NO}_{3}\right)_{2}$

$M_{r}=443.01$

Triclinic, $P \overline{1}$

$a=6.2620(5) \AA$

$b=7.1053(7) \AA$

$c=10.7101(10) \AA$

$\alpha=102.461(8)^{\circ}$

$\beta=96.754(7)^{\circ}$

$\gamma=114.823(8)$

$V=410.49(7) \AA^{3}$

$Z=1$

Mo $K \alpha$ radiation

$\mu=1.26 \mathrm{~mm}^{-1}$

$T=100 \mathrm{~K}$

$0.08 \times 0.07 \times 0.06 \mathrm{~mm}$

\subsection{Data collection}

Bruker SMART 1K CCD areadetector diffractometer

Absorption correction: analytical (CrysAlis RED; Oxford Diffraction, 2003)

$T_{\min }=0.888, T_{\max }=0.936$

2781 measured reflections 1801 independent reflections 1654 reflections with $I>2 \sigma(I)$ $R_{\text {int }}=0.020$

\subsection{Refinement}

$R\left[F^{2}>2 \sigma\left(F^{2}\right)\right]=0.032$

$w R\left(F^{2}\right)=0.070$

$S=1.06$

1801 reflections

146 parameters

$\mathrm{H}$ atoms treated by a mixture of independent and constrained refinement

$\Delta \rho_{\max }=0.45$ e $\AA^{-3}$

$\Delta \rho_{\min }=-0.39 \mathrm{e}^{-3}$

Table 1

Selected bond lengths (

\begin{tabular}{llll}
\hline $\mathrm{Ni} 1-\mathrm{O} 1 W$ & $2.0184(16)$ & $\mathrm{Ni} 1-\mathrm{O} 2 W$ & $2.0990(16)$ \\
$\mathrm{Ni1}-\mathrm{O} 3 W$ & $2.0242(16)$ & & \\
\hline
\end{tabular}

Table 2

Hydrogen-bond geometry $\left(\AA,{ }^{\circ}\right)$.

\begin{tabular}{lllll}
\hline$D-\mathrm{H} \cdots A$ & $D-\mathrm{H}$ & $\mathrm{H} \cdots A$ & $D \cdots A$ & $D-\mathrm{H} \cdots A$ \\
\hline $\mathrm{N} 1-\mathrm{H} 1 \cdots \mathrm{O} 2 W$ & $0.87(2)$ & $2.06(3)$ & $2.906(2)$ & $167(2)$ \\
$\mathrm{O} 1 W-\mathrm{H} 11 W \cdots \mathrm{O} 71^{\mathrm{i}}$ & $0.77(3)$ & $1.85(3)$ & $2.612(2)$ & $173(3)$ \\
$\mathrm{O} 1 W-\mathrm{H} 12 W \cdots \mathrm{O} 72^{\mathrm{ii}}$ & $0.78(3)$ & $1.97(3)$ & $2.748(2)$ & $173(3)$ \\
$\mathrm{O} 2 W-\mathrm{H} 21 W \cdots \mathrm{O} 2^{\mathrm{iii}}$ & $0.88(3)$ & $1.90(3)$ & $2.772(2)$ & $173(2)$ \\
$\mathrm{O} 2 W-\mathrm{H} 22 W \cdots \mathrm{O} 2^{\mathrm{iv}}$ & $0.77(3)$ & $1.98(3)$ & $2.743(2)$ & $169(3)$ \\
$\mathrm{O} 3 W-\mathrm{H} 31 W \cdots \mathrm{O} 72^{\mathrm{i}}$ & $0.74(3)$ & $1.92(3)$ & $2.660(2)$ & $174(3)$ \\
$\mathrm{O} 3 W-\mathrm{H} 32 W \cdots \mathrm{O} 2^{\mathrm{v}}$ & $0.81(3)$ & $2.01(3)$ & $2.813(2)$ & $172(3)$
\end{tabular}

Symmetry codes: (i) $-x+1,-y,-z+2$; (ii) $\quad-x+2,-y,-z+2$; $-x+1,-y-1,-z+1$; (iv) $x-1, y, z ;$ (v) $-x+1,-y,-z+1$.

Data collection: CrysAlis PRO (Agilent, 2012); cell refinement: CrysAlis PRO; data reduction: CrysAlis PRO; program(s) used to solve structure: SIR92 (Altomare et al., 1993); program(s) used to refine structure: SHELXL97 (Sheldrick, 2008); molecular graphics: 
ORTEP-3 for Windows (Farrugia, 2012); software used to prepare material for publication: Win $G X$ (Farrugia, 2012).

\section{Acknowledgements}

This work has been funded by Eusko Jaurlaritza/Gobierno Vasco (Grant IT477-10), Universidad del País Vasco/Euskal Herriko Unibertsitatea (EHUA14/09, Grant UFI11/53), and the Ministerio de Economía y Competitividad (MAT201346502-C2-1-P). The authors are thankful for technical and human support provided by S. GIker of UPV/EHU.

Supporting information for this paper is available from the IUCr electronic archives (Reference: WM5241).

\section{References}

Agilent (2012). CrysAlis PRO. Agilent Technologies Ltd, Yarnton, England. Altomare, A., Cascarano, G., Giacovazzo, C. \& Guagliardi, A. (1993). J. Appl. Cryst. 26, 343-350.

Farrugia, L. J. (2012). J. Appl. Cryst. 45, 849-854.

Oxford Diffraction (2003). CrysAlis RED. Oxford Diffraction Ltd, Abingdon, England.

Sheldrick, G. M. (2008). Acta Cryst. A64, 112-122.

Song, Y.-S., Yan, B. \& Chen, Z.-X. (2005). Inorg. Chem. Commun. 8, 11651168.

Zeng, Y.-F., Zhao, J.-P., Hu, B.-W., Hu, X., Liu, F.-C., Ribas, J., Ribas-Ariño, J. \& Bu, X. H. (2007). Chem. Eur. J. 13, 9924-9930.

Zhang, X.-L., Lu, Y.-J., Li, J.-Z. \& Ng, S. W. (2005). Acta Cryst. E61, m1063m1064.

Zhang, X.-L. \& Ng, S. W. (2005a). Acta Cryst. E61, m1140-m1141.

Zhang, X.-L. \& Ng, S. W. (2005b). Acta Cryst. E61, m1142-m1143. 


\section{supporting information}

Acta Cryst. (2015). E71, m238-m239 [https://doi.org/10.1107/S2056989015022422]

The crystal structure of a new polymorph of hexaaquanickel(II) bis(6-oxo-1,6dihydropyridine-3-carboxylate)

Rubén Pérez-Aguirre, Sonia Pérez-Yáñez, Garikoitz Beobide, Oscar Castillo, Juan Manuel Gutiérrez-Zorrilla and Antonio Luque

\section{S1. Experimental}

6-Oxo-1,6-dihydropyridine-3-carboxylic acid $(0.8 \mathrm{mmol})$ and $\mathrm{Ni}\left(\mathrm{NO}_{3}\right)_{2} \cdot 6 \mathrm{H}_{2} \mathrm{O}(0.4 \mathrm{mmol})$ were dissolved in $40 \mathrm{ml}$ of distilled water. After stirring for half an hour, the solution was left evaporating at room temperature. Two weeks later light green crystals of the title compound were obtained.

\section{S2. Refinement}

$\mathrm{H}$ atoms bonded to $\mathrm{N}$ and $\mathrm{O}$ atoms were located in a difference map and were refined with $U_{\text {iso }}(\mathrm{H})=1.2 U_{\text {eq }}(\mathrm{N})$ and $U_{\text {iso }}(\mathrm{H})=1.5 U_{\mathrm{eq}}(\mathrm{O})$. Other $\mathrm{H}$ atoms were positioned geometrically and refined using a riding model with $\mathrm{C}-\mathrm{H}=0.93 \AA$ and with $U_{\mathrm{iso}}(\mathrm{H})=1.2 U_{\mathrm{eq}}(\mathrm{C})$.

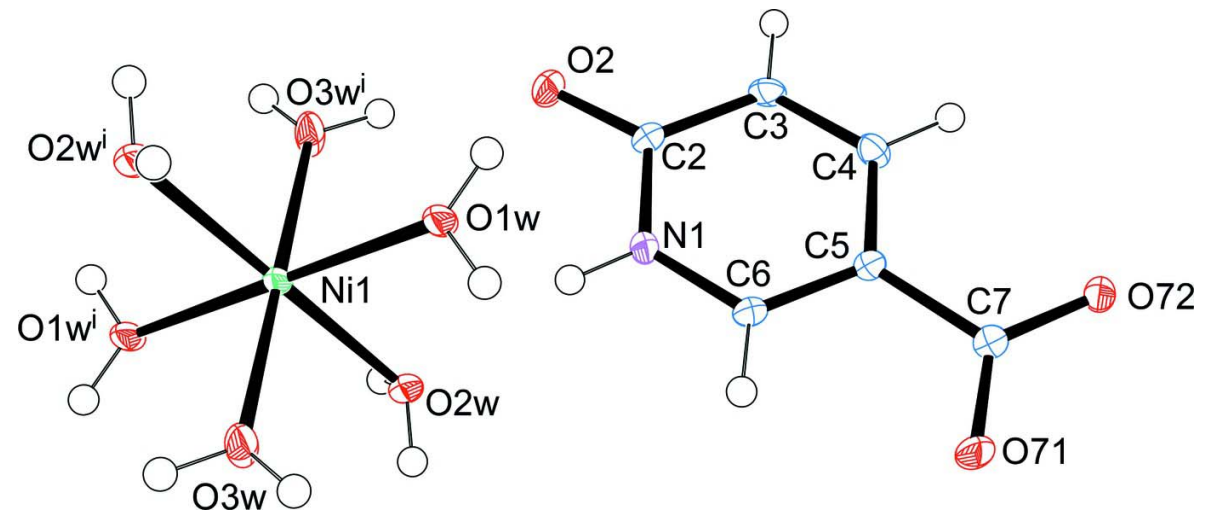

Figure 1

The structures of the molecular entities in (I), drawn with displacement ellipsoids at the $50 \%$ probability level.

[Symmetry code: $-x+1,-y,-z+1$.] 


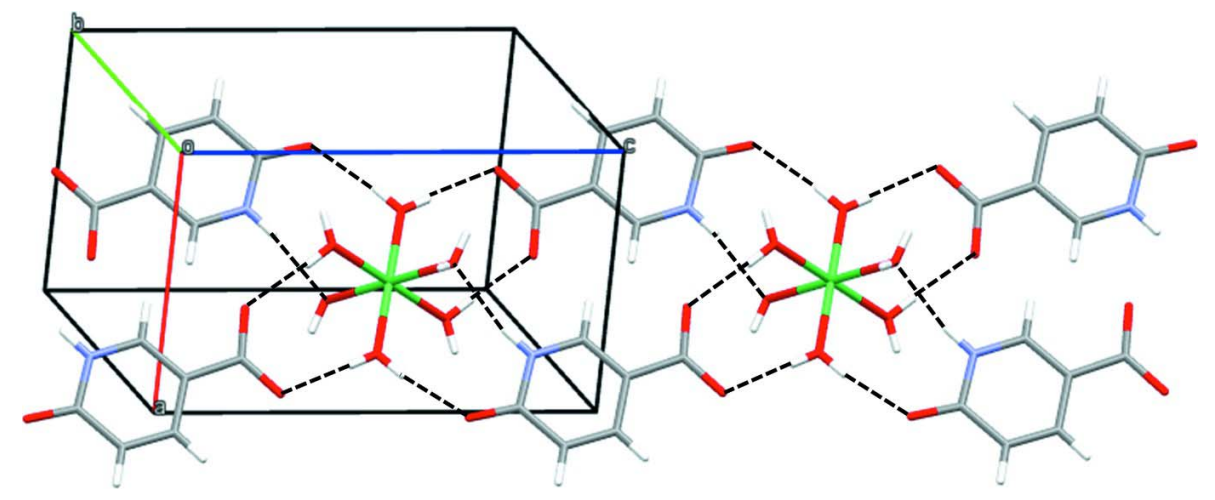

Figure 2

Hydrogen-bonding interactions (dashed lines) taking place between the $\left[\mathrm{Ni}\left(\mathrm{H}_{2} \mathrm{O}\right)_{6}\right]^{2+}$ complex cations and the 6-oxo-1,6dihydropyridine-3-carboxylate anions.

Hexaaquanickel(II) bis(6-oxo-1,6-dihydropyridine-3-carboxylate)

\section{Crystal data}

$\left[\mathrm{Ni}\left(\mathrm{H}_{2} \mathrm{O}\right)_{6}\right]\left(\mathrm{C}_{6} \mathrm{H}_{4} \mathrm{NO}_{3}\right)_{2}$

$$
M_{r}=443.01
$$

Triclinic, $P \overline{1}$

$a=6.2620(5) \AA$

$b=7.1053(7) \AA$

$c=10.7101(10) \AA$

$\alpha=102.461(8)^{\circ}$

$\beta=96.754(7)^{\circ}$

$\gamma=114.823(8)^{\circ}$

$V=410.49(7) \AA^{3}$

\section{Data collection}

Bruker SMART 1K CCD area-detector diffractometer

Radiation source: sealed tube

Graphite monochromator

Detector resolution: 8.192 pixels $\mathrm{mm}^{-1}$

thin-slice $\omega$ scans

Absorption correction: analytical

(CrysAlis RED; Oxford Diffraction, 2003)

$T_{\min }=0.888, T_{\max }=0.936$

\section{Refinement}

Refinement on $F^{2}$

Least-squares matrix: full

$R\left[F^{2}>2 \sigma\left(F^{2}\right)\right]=0.032$

$w R\left(F^{2}\right)=0.070$

$S=1.06$

1801 reflections

146 parameters

0 restraints

Primary atom site location: structure-invariant direct methods
$Z=1$

$F(000)=230$

$D_{\mathrm{x}}=1.792 \mathrm{Mg} \mathrm{m}^{-3}$

Mo $K \alpha$ radiation, $\lambda=0.71073 \AA$

Cell parameters from 2781 reflections

$\theta=2.0-28.2^{\circ}$

$\mu=1.26 \mathrm{~mm}^{-1}$

$T=100 \mathrm{~K}$

Block, light green

$0.08 \times 0.07 \times 0.06 \mathrm{~mm}$

2781 measured reflections

1801 independent reflections

1654 reflections with $I>2 \sigma(I)$

$R_{\text {int }}=0.020$

$\theta_{\max }=28.2^{\circ}, \theta_{\min }=2.0^{\circ}$

$h=-5 \rightarrow 8$

$k=-9 \rightarrow 5$

$l=-14 \rightarrow 13$

Secondary atom site location: difference Fourier map

Hydrogen site location: mixed

$\mathrm{H}$ atoms treated by a mixture of independent and constrained refinement

$w=1 /\left[\sigma^{2}\left(F_{\mathrm{o}}^{2}\right)+(0.0191 P)^{2}+0.3293 P\right]$

where $P=\left(F_{\mathrm{o}}{ }^{2}+2 F_{\mathrm{c}}{ }^{2}\right) / 3$

$(\Delta / \sigma)_{\max }<0.001$

$\Delta \rho_{\max }=0.45 \mathrm{e} \AA^{-3}$

$\Delta \rho_{\min }=-0.39$ e $\AA^{-3}$ 


\section{Special details}

Refinement. Refinement of $\mathrm{F}^{2}$ against ALL reflections. The weighted R-factor $\mathrm{wR}$ and goodness of fit $\mathrm{S}$ are based on $\mathrm{F}^{2}$, conventional R-factors $R$ are based on $F$, with $F$ set to zero for negative $F^{2}$. The threshold expression of $F^{2}>2 \operatorname{sigma}\left(F^{2}\right)$ is used only for calculating R-factors(gt) etc. and is not relevant to the choice of reflections for refinement. R-factors based on $\mathrm{F}^{2}$ are statistically about twice as large as those based on F, and R- factors based on ALL data will be even larger.

Fractional atomic coordinates and isotropic or equivalent isotropic displacement parameters $\left(\AA^{2}\right)$

\begin{tabular}{lllll}
\hline & $x$ & $y$ & $z$ & $U_{\text {iso }} / U_{\text {eq }}$ \\
\hline Ni1 & 0.5000 & 0.0000 & 0.5000 & $0.01281(12)$ \\
O1W & $0.7185(3)$ & $0.1609(3)$ & $0.68193(15)$ & $0.0168(3)$ \\
O71 & $0.5271(3)$ & $-0.1565(3)$ & $1.13681(15)$ & $0.0202(4)$ \\
O2W & $0.3038(3)$ & $-0.2654(3)$ & $0.56622(15)$ & $0.0154(3)$ \\
O3W & $0.2933(3)$ & $0.1461(3)$ & $0.55076(17)$ & $0.0231(4)$ \\
O2 & $0.8636(3)$ & $-0.3362(3)$ & $0.62046(14)$ & $0.0176(3)$ \\
O72 & $0.8231(3)$ & $-0.2245(3)$ & $1.21937(15)$ & $0.0202(4)$ \\
N1 & $0.6616(3)$ & $-0.2558(3)$ & $0.76999(18)$ & $0.0162(4)$ \\
C2 & $0.8355(4)$ & $-0.3128(4)$ & $0.7379(2)$ & $0.0141(4)$ \\
C4 & $0.9331(4)$ & $-0.3021(3)$ & $0.9661(2)$ & $0.0140(4)$ \\
H4 & 1.0278 & -0.3170 & 1.0333 & $0.017^{*}$ \\
C3 & $0.9726(4)$ & $-0.3378(4)$ & $0.8423(2)$ & $0.0148(4)$ \\
H3 & 1.0917 & -0.3793 & 0.8264 & $0.018^{*}$ \\
C5 & $0.7512(4)$ & $-0.2432(3)$ & $0.9937(2)$ & $0.0130(4)$ \\
C7 & $0.6965(4)$ & $-0.2048(3)$ & $1.1271(2)$ & $0.0144(4)$ \\
C6 & $0.6196(4)$ & $-0.2203(4)$ & $0.8921(2)$ & $0.0154(4)$ \\
H6 & 0.4995 & -0.1797 & 0.9070 & $0.018^{*}$ \\
H1 & $0.574(4)$ & $-0.240(4)$ & $0.708(3)$ & $0.018^{*}$ \\
H31W & $0.266(5)$ & $0.177(4)$ & $0.615(3)$ & $0.023^{*}$ \\
H21W & $0.262(5)$ & $-0.387(5)$ & $0.505(3)$ & $0.023^{*}$ \\
H22W & $0.189(5)$ & $-0.270(4)$ & $0.588(3)$ & $0.023^{*}$ \\
H11W & $0.650(5)$ & $0.171(4)$ & $0.736(3)$ & $0.023^{*}$ \\
H12W & $0.844(5)$ & $0.170(4)$ & $0.712(3)$ & $0.023^{*}$ \\
H32W & $0.236(5)$ & $0.196(5)$ & $0.503(3)$ & $0.038(9) *$ \\
& & & &
\end{tabular}

Atomic displacement parameters $\left(\AA^{2}\right)$

\begin{tabular}{lllllll}
\hline & $U^{11}$ & $U^{22}$ & $U^{33}$ & $U^{12}$ & $U^{13}$ & $U^{23}$ \\
\hline Ni1 & $0.0108(2)$ & $0.0231(2)$ & $0.0093(2)$ & $0.01104(17)$ & $0.00397(15)$ & $0.00593(16)$ \\
O1W & $0.0119(8)$ & $0.0321(10)$ & $0.0095(8)$ & $0.0130(7)$ & $0.0037(6)$ & $0.0053(7)$ \\
O71 & $0.0201(8)$ & $0.0353(10)$ & $0.0169(8)$ & $0.0204(8)$ & $0.0102(7)$ & $0.0109(7)$ \\
O2W & $0.0127(8)$ & $0.0239(9)$ & $0.0126(8)$ & $0.0102(7)$ & $0.0056(6)$ & $0.0058(7)$ \\
O3W & $0.0303(10)$ & $0.0467(12)$ & $0.0121(8)$ & $0.0320(9)$ & $0.0110(7)$ & $0.0135(8)$ \\
O2 & $0.0198(8)$ & $0.0248(9)$ & $0.0126(8)$ & $0.0127(7)$ & $0.0073(6)$ & $0.0067(7)$ \\
O72 & $0.0180(8)$ & $0.0384(10)$ & $0.0112(8)$ & $0.0187(8)$ & $0.0048(6)$ & $0.0079(7)$ \\
N1 & $0.0152(9)$ & $0.0275(11)$ & $0.0121(9)$ & $0.0135(8)$ & $0.0047(8)$ & $0.0094(8)$ \\
C2 & $0.0133(10)$ & $0.0176(11)$ & $0.0130(10)$ & $0.0074(9)$ & $0.0061(8)$ & $0.0054(9)$ \\
C4 & $0.0132(10)$ & $0.0156(11)$ & $0.0138(10)$ & $0.0084(9)$ & $0.0010(8)$ & $0.0033(9)$ \\
C3 & $0.0118(10)$ & $0.0190(11)$ & $0.0170(11)$ & $0.0102(9)$ & $0.0053(8)$ & $0.0042(9)$
\end{tabular}




\begin{tabular}{lllllll} 
C5 & $0.0113(10)$ & $0.0159(11)$ & $0.0115(10)$ & $0.0058(8)$ & $0.0038(8)$ & $0.0038(8)$ \\
C7 & $0.0127(10)$ & $0.0169(11)$ & $0.0133(11)$ & $0.0062(9)$ & $0.0039(8)$ & $0.0047(9)$ \\
C6 & $0.0130(10)$ & $0.0233(12)$ & $0.0145(11)$ & $0.0107(9)$ & $0.0066(9)$ & $0.0075(9)$ \\
\hline
\end{tabular}

Geometric parameters $\left(\AA,{ }^{\circ}\right)$

\begin{tabular}{|c|c|c|c|}
\hline Ni1-O1W & $2.0184(16)$ & $\mathrm{O} 2-\mathrm{C} 2$ & $1.275(2)$ \\
\hline $\mathrm{Ni} 1-\mathrm{O} 1 \mathrm{~W}^{\mathrm{i}}$ & $2.0184(16)$ & $\mathrm{O} 72-\mathrm{C} 7$ & $1.262(3)$ \\
\hline $\mathrm{Ni} 1-\mathrm{O} 3 \mathrm{~W}^{\mathrm{i}}$ & $2.0242(16)$ & $\mathrm{N} 1-\mathrm{C} 6$ & $1.356(3)$ \\
\hline $\mathrm{Ni1}-\mathrm{O} 3 \mathrm{~W}$ & $2.0242(16)$ & $\mathrm{N} 1-\mathrm{C} 2$ & $1.366(3)$ \\
\hline $\mathrm{Ni} 1-\mathrm{O} 2 \mathrm{~W}^{\mathrm{i}}$ & $2.0990(16)$ & $\mathrm{N} 1-\mathrm{H} 1$ & $0.87(2)$ \\
\hline $\mathrm{Nil}-\mathrm{O} 2 \mathrm{~W}$ & $2.0990(16)$ & $\mathrm{C} 2-\mathrm{C} 3$ & $1.417(3)$ \\
\hline $\mathrm{O} 1 \mathrm{~W}-\mathrm{H} 11 \mathrm{~W}$ & $0.77(3)$ & $\mathrm{C} 4-\mathrm{C} 3$ & $1.367(3)$ \\
\hline $\mathrm{O} 1 \mathrm{~W}-\mathrm{H} 12 \mathrm{~W}$ & $0.78(3)$ & $\mathrm{C} 4-\mathrm{C} 5$ & $1.409(3)$ \\
\hline $\mathrm{O} 71-\mathrm{C} 7$ & $1.253(2)$ & $\mathrm{C} 4-\mathrm{H} 4$ & 0.9300 \\
\hline $\mathrm{O} 2 \mathrm{~W}-\mathrm{H} 21 \mathrm{~W}$ & $0.88(3)$ & $\mathrm{C} 3-\mathrm{H} 3$ & 0.9300 \\
\hline $\mathrm{O} 2 \mathrm{~W}-\mathrm{H} 22 \mathrm{~W}$ & $0.77(3)$ & $\mathrm{C} 5-\mathrm{C} 6$ & $1.366(3)$ \\
\hline $\mathrm{O} 3 \mathrm{~W}-\mathrm{H} 31 \mathrm{~W}$ & $0.74(3)$ & $\mathrm{C} 5-\mathrm{C} 7$ & $1.503(3)$ \\
\hline $\mathrm{O} 3 \mathrm{~W}-\mathrm{H} 32 \mathrm{~W}$ & $0.81(3)$ & $\mathrm{C} 6-\mathrm{H} 6$ & 0.9300 \\
\hline $\mathrm{O} 1 \mathrm{~W}-\mathrm{Ni} 1-\mathrm{O} 1 \mathrm{~W}^{\mathrm{i}}$ & 180.0 & $\mathrm{H} 31 \mathrm{~W}-\mathrm{O} 3 \mathrm{~W}-\mathrm{H} 32 \mathrm{~W}$ & $106(3)$ \\
\hline $\mathrm{O} 1 \mathrm{~W}-\mathrm{Ni1}-\mathrm{O} 2 \mathrm{~W}$ & $89.95(6)$ & $\mathrm{C} 6-\mathrm{N} 1-\mathrm{C} 2$ & $124.28(18)$ \\
\hline $\mathrm{O} 1 \mathrm{~W}-\mathrm{Ni} 1-\mathrm{O} 2 \mathrm{~W}^{\mathrm{i}}$ & $90.05(6)$ & $\mathrm{C} 6-\mathrm{N} 1-\mathrm{H} 1$ & $118.1(16)$ \\
\hline $\mathrm{O} 1 \mathrm{~W}-\mathrm{Ni} 1-\mathrm{O} 3 \mathrm{~W}$ & $88.06(7)$ & $\mathrm{C} 2-\mathrm{N} 1-\mathrm{H} 1$ & $117.6(16)$ \\
\hline $\mathrm{O} 1 \mathrm{~W}-\mathrm{Ni} 1-\mathrm{O} 3 \mathrm{~W}^{\mathrm{i}}$ & $91.94(7)$ & $\mathrm{O} 2-\mathrm{C} 2-\mathrm{N} 1$ & $118.94(19)$ \\
\hline $\mathrm{O} 2 \mathrm{~W}-\mathrm{Ni1}-\mathrm{O} 3 \mathrm{~W}$ & $92.99(7)$ & $\mathrm{O} 2-\mathrm{C} 2-\mathrm{C} 3$ & $125.81(19)$ \\
\hline $\mathrm{O} 2 \mathrm{~W}-\mathrm{Ni} 1-\mathrm{O} 3 \mathrm{~W}^{\mathrm{i}}$ & $87.01(7)$ & $\mathrm{N} 1-\mathrm{C} 2-\mathrm{C} 3$ & $115.24(18)$ \\
\hline $\mathrm{O} 1 \mathrm{~W}^{\mathrm{i}}-\mathrm{Ni1}-\mathrm{O} 3 \mathrm{~W}^{\mathrm{i}}$ & $88.06(7)$ & $\mathrm{C} 3-\mathrm{C} 4-\mathrm{C} 5$ & $121.08(19)$ \\
\hline $\mathrm{O} 1 \mathrm{~W}-\mathrm{Ni1}-\mathrm{O} 3 \mathrm{~W}$ & $91.94(7)$ & $\mathrm{C} 3-\mathrm{C} 4-\mathrm{H} 4$ & 119.5 \\
\hline $\mathrm{O} 3 \mathrm{~W}^{\mathrm{i}}-\mathrm{Ni} 1-\mathrm{O} 3 \mathrm{~W}$ & 180.0 & $\mathrm{C} 5-\mathrm{C} 4-\mathrm{H} 4$ & 119.5 \\
\hline $\mathrm{O} 1 \mathrm{~W}^{\mathrm{i}}-\mathrm{Ni} 1-\mathrm{O} 2 \mathrm{~W}^{\mathrm{i}}$ & $89.95(6)$ & $\mathrm{C} 4-\mathrm{C} 3-\mathrm{C} 2$ & $121.22(19)$ \\
\hline $\mathrm{O} 3 \mathrm{~W}^{\mathrm{i}}-\mathrm{Ni1}-\mathrm{O} 2 \mathrm{~W}^{\mathrm{i}}$ & $92.99(7)$ & $\mathrm{C} 4-\mathrm{C} 3-\mathrm{H} 3$ & 119.4 \\
\hline $\mathrm{O} 1 \mathrm{~W}-\mathrm{Ni} 1-\mathrm{O} 2 \mathrm{~W}$ & $90.05(6)$ & $\mathrm{C} 2-\mathrm{C} 3-\mathrm{H} 3$ & 119.4 \\
\hline $\mathrm{O} 3 \mathrm{~W}^{\mathrm{i}}-\mathrm{Ni} 1-\mathrm{O} 2 \mathrm{~W}$ & $87.01(7)$ & $\mathrm{C} 6-\mathrm{C} 5-\mathrm{C} 4$ & $117.18(19)$ \\
\hline $\mathrm{O} 2 \mathrm{~W}^{\mathrm{i}}-\mathrm{Ni} 1-\mathrm{O} 2 \mathrm{~W}$ & 180.0 & $\mathrm{C} 6-\mathrm{C} 5-\mathrm{C} 7$ & $119.20(18)$ \\
\hline Ni1-O1W-H11W & $114(2)$ & $\mathrm{C} 4-\mathrm{C} 5-\mathrm{C} 7$ & $123.62(18)$ \\
\hline $\mathrm{Ni1}-\mathrm{O} 1 \mathrm{~W}-\mathrm{H} 12 \mathrm{~W}$ & $130(2)$ & $\mathrm{O} 71-\mathrm{C} 7-\mathrm{O} 72$ & $125.51(19)$ \\
\hline $\mathrm{H} 11 \mathrm{~W}-\mathrm{O} 1 \mathrm{~W}-\mathrm{H} 12 \mathrm{~W}$ & $110(3)$ & $\mathrm{O} 71-\mathrm{C} 7-\mathrm{C} 5$ & $116.72(18)$ \\
\hline $\mathrm{Ni} 1-\mathrm{O} 2 \mathrm{~W}-\mathrm{H} 21 \mathrm{~W}$ & $110.1(17)$ & $\mathrm{O} 72-\mathrm{C} 7-\mathrm{C} 5$ & $117.77(18)$ \\
\hline $\mathrm{Ni} 1-\mathrm{O} 2 \mathrm{~W}-\mathrm{H} 22 \mathrm{~W}$ & $116(2)$ & $\mathrm{N} 1-\mathrm{C} 6-\mathrm{C} 5$ & $120.98(19)$ \\
\hline $\mathrm{H} 21 \mathrm{~W}-\mathrm{O} 2 \mathrm{~W}-\mathrm{H} 22 \mathrm{~W}$ & $108(3)$ & $\mathrm{N} 1-\mathrm{C} 6-\mathrm{H} 6$ & 119.5 \\
\hline $\mathrm{Ni1}-\mathrm{O} 3 \mathrm{~W}-\mathrm{H} 31 \mathrm{~W}$ & $129(2)$ & $\mathrm{C} 5-\mathrm{C} 6-\mathrm{H} 6$ & 119.5 \\
\hline $\mathrm{Ni1}-\mathrm{O} 3 \mathrm{~W}-\mathrm{H} 32 \mathrm{~W}$ & $124(2)$ & & \\
\hline $\mathrm{C} 6-\mathrm{N} 1-\mathrm{C} 2-\mathrm{O} 2$ & $-178.1(2)$ & $\mathrm{C} 6-\mathrm{C} 5-\mathrm{C} 7-\mathrm{O} 71$ & $1.2(3)$ \\
\hline $\mathrm{C} 6-\mathrm{N} 1-\mathrm{C} 2-\mathrm{C} 3$ & $1.1(3)$ & $\mathrm{C} 4-\mathrm{C} 5-\mathrm{C} 7-\mathrm{O} 71$ & $-178.9(2)$ \\
\hline $\mathrm{C} 5-\mathrm{C} 4-\mathrm{C} 3-\mathrm{C} 2$ & $1.2(3)$ & $\mathrm{C} 6-\mathrm{C} 5-\mathrm{C} 7-\mathrm{O} 72$ & $-179.1(2)$ \\
\hline $\mathrm{O} 2-\mathrm{C} 2-\mathrm{C} 3-\mathrm{C} 4$ & $177.9(2)$ & $\mathrm{C} 4-\mathrm{C} 5-\mathrm{C} 7-\mathrm{O} 72$ & $0.8(3)$ \\
\hline
\end{tabular}




\begin{tabular}{llll}
$\mathrm{N} 1-\mathrm{C} 2-\mathrm{C} 3-\mathrm{C} 4$ & $-1.2(3)$ & $\mathrm{C} 2-\mathrm{N} 1-\mathrm{C} 6-\mathrm{C} 5$ & $-1.0(3)$ \\
$\mathrm{C} 3-\mathrm{C} 4-\mathrm{C} 5-\mathrm{C} 6$ & $-1.0(3)$ & $\mathrm{C} 4-\mathrm{C} 5-\mathrm{C} 6-\mathrm{N} 1$ & $0.8(3)$ \\
$\mathrm{C} 3-\mathrm{C} 4-\mathrm{C} 5-\mathrm{C} 7$ & $179.1(2)$ & $\mathrm{C} 7-\mathrm{C} 5-\mathrm{C} 6-\mathrm{N} 1$ & $-179.3(2)$ \\
\hline
\end{tabular}

Symmetry code: (i) $-x+1,-y,-z+1$.

Hydrogen-bond geometry $\left(A,{ }^{\circ}\right)$

\begin{tabular}{lllll}
\hline$D-\mathrm{H} \cdots A$ & $D-\mathrm{H}$ & $\mathrm{H} \cdots A$ & $D \cdots A$ & $D-\mathrm{H} \cdots A$ \\
\hline $\mathrm{N} 1-\mathrm{H} 1 \cdots \mathrm{O} 2 W$ & $0.87(2)$ & $2.06(3)$ & $2.906(2)$ & $167(2)$ \\
$\mathrm{O} 1 W-\mathrm{H} 11 W \cdots \mathrm{O} 71^{\mathrm{ii}}$ & $0.77(3)$ & $1.85(3)$ & $2.612(2)$ & $173(3)$ \\
$\mathrm{O} 1 W-\mathrm{H} 12 W \cdots \mathrm{O} 72^{\mathrm{iii}}$ & $0.78(3)$ & $1.97(3)$ & $2.748(2)$ & $173(3)$ \\
$\mathrm{O} 2 W-\mathrm{H} 21 W \cdots \mathrm{O} 2^{\mathrm{iv}}$ & $0.88(3)$ & $1.90(3)$ & $2.772(2)$ & $173(2)$ \\
$\mathrm{O} 2 W-\mathrm{H} 22 W \cdots \mathrm{O} 2^{v}$ & $0.77(3)$ & $1.98(3)$ & $2.743(2)$ & $169(3)$ \\
$\mathrm{O} 3 W-\mathrm{H} 31 W \cdots \mathrm{O} 72^{\mathrm{ii}}$ & $0.74(3)$ & $1.92(3)$ & $2.660(2)$ & $174(3)$ \\
$\mathrm{O} 3 W-\mathrm{H} 32 W \cdots \mathrm{O} 2^{\mathrm{i}}$ & $0.81(3)$ & $2.01(3)$ & $2.813(2)$ & $172(3)$
\end{tabular}

Symmetry codes: (i) $-x+1,-y,-z+1$; (ii) $-x+1,-y,-z+2$; (iii) $-x+2,-y,-z+2$; (iv) $-x+1,-y-1,-z+1$; (v) $x-1, y, z$. 